PROCEEDINGS OF THE

AMERICAN MATHEMATICAL SOCIETY

Volume 136, Number 7, July 2008, Pages 2289-2297

S 0002-9939(08)09271-X

Article electronically published on March 19, 2008

\title{
ON THE PLANE SECTION OF AN INTEGRAL CURVE IN POSITIVE CHARACTERISTIC
}

\author{
PAOLA BONACINI \\ (Communicated by Ted Chinburg)
}

\begin{abstract}
If $C \subset \mathbb{P}_{k}^{3}$ is an integral curve and $k$ an algebraically closed field of characteristic 0 , it is known that the points of the general plane section $C \cap H$ of $C$ are in uniform position. From this it follows easily that the general minimal curve containing $C \cap H$ is irreducible. If chark $=p>0$, the points of $C \cap H$ may not be in uniform position. However, we prove that the general minimal curve containing $C \cap H$ is still irreducible.
\end{abstract}

\section{INTRODUCTION}

Let $C$ be an integral curve in $\mathbb{P}_{k}^{3}$, where $k$ is an algebraically closed field of characteristic 0. Harris in [7] proves that the points of a general plane section $C \cap H$ of $C$ lie in uniform position, which means that all the subsets of $C \cap H$ of the same cardinality have the same Hilbert function. A set of points with the Uniform Position Property has some interesting properties, as the fact that the general minimal curve containing it is irreducible.

In positive characteristic the Uniform Position Property is not satisfied for all the generic plane sections of integral curves. Indeed in [14, Example 1.2] Rathmann gives an example of a complete intersection integral curve, whose generic plane section is not in uniform position. However the general minimal curve containing it is still irreducible.

In $\S 3$ we prove that, independent of the characteristic of the base field, the general minimal curve containing the generic plane section of an integral curve is always irreducible. From it there also easily follows the fact that the generic plane section of an integral curve has the Hilbert function of decreasing type, a fact well known in characteristic 0 (see [7] for example) and proved in positive characteristic in 1 .

\section{Preliminaries And nOtation}

Let us denote $X$ as a finite set of points in $\mathbb{P}_{k}^{2}$, where $k$ is an algebraically closed field. Let us recall the following:

Definition 2.1. We say that $X$ has the Uniform Position Property (briefly UPP) if, for any $n \leq \operatorname{deg} X$, all the subsets of $n$ points have the same Hilbert function.

Received by the editors July 26, 2006, and, in revised form, February 19, 2007.

2000 Mathematics Subject Classification. Primary 14H50; Secondary 13D40.

Key words and phrases. Integral curve, plane section, minimal curve, positive characteristic.

(C)2008 American Mathematical Society

Reverts to public domain 28 years from publication 
If this happens, then (see [3]) the Hilbert function of a set $Z \subset X$ of $n$ points is the following truncated function:

$$
H(Z, i)=\min \{H(X, i), n\} \forall i
$$

One of the main consequences of the Uniform Position Property is the following:

Proposition 2.2 ([7, p. 198]). If $X$ is a set of points in $\mathbb{P}^{2}$ in uniform position and $X^{\prime} \subset X$ is such that $h^{1}\left(\mathscr{I}_{X^{\prime}}(l)\right) \neq 0$, then $h^{0}\left(\mathscr{I}_{X}(l)\right)=h^{0}\left(\mathscr{I}_{X^{\prime}}(l)\right)$. So every curve of degree l containing $X^{\prime}$ contains $X$. In particular this holds when $\operatorname{deg} X^{\prime}=\left(\begin{array}{c}l+2 \\ 2\end{array}\right)$.

Proof. If $h^{1}\left(\mathscr{I}_{X^{\prime}}(l)\right) \neq 0$, then

$$
H\left(X^{\prime}, l\right)=h^{0}\left(\mathscr{O}_{\mathbb{P}^{2}}(l)\right)-h^{0}\left(\mathscr{I}_{X^{\prime}}(l)\right)<\operatorname{deg} X^{\prime},
$$

and so, by (2.1), $H\left(X^{\prime}, l\right)=H(X, l)$, which implies that

$$
h^{0}\left(\mathscr{I}_{X^{\prime}}(l)\right)=h^{0}\left(\mathscr{I}_{X}(l)\right) .
$$

If $\operatorname{deg} X^{\prime}=\left(\begin{array}{c}l+2 \\ 2\end{array}\right)$ and $h^{0}\left(\mathscr{I}_{X^{\prime}}(l)\right) \neq 0$, one has

$$
\chi\left(\mathscr{I}_{X}(l)\right)=\chi\left(\mathscr{O}_{\mathbb{P}^{2}}(l)\right)-\chi\left(\mathscr{O}_{X}(l)\right)=0,
$$

so that

$$
h^{1}\left(\mathscr{I}_{X^{\prime}}(l)\right)=h^{0}\left(\mathscr{I}_{X^{\prime}}(l)\right) \neq 0 .
$$

If $h^{0}\left(\mathscr{I}_{X^{\prime}}(l)\right)=0$, then it must be $h^{0}\left(\mathscr{I}_{X}(l)\right)=0$.

An important corollary of this fact is:

Proposition 2.3 ([10, Remark 1.2(ii)], [2, Theorem 3.4]). Let $X \subset \mathbb{P}^{2}$ be a reduced scheme in uniform position such that

$$
\operatorname{deg} X=n=\left(\begin{array}{c}
d+2 \\
2
\end{array}\right)+h
$$

with $0 \leq h \leq d+1$. If

$$
g=\inf \left\{i \in \mathbb{N} \mid H^{0}\left(\mathscr{I}_{X}(i)\right) \neq 0\right\}
$$

then:

(1) if either $g \leq d$ or $g=d+1$ and $h \geq 2$, every element of $H^{0}\left(\mathscr{I}_{X}(g)\right)$ is irreducible;

(2) if $g=d+1$ and $h=0,1$, the generic element of $H^{0}\left(\mathscr{I}_{X}(g)\right)$ is irreducible. In particular there exists a minimal smooth curve containing $X$.

Proof. We follow the proof in [10. First of all note that $h^{0}\left(\mathscr{I}_{X}(d+1)\right) \neq 0$, so that $g \leq d+1$. Indeed

$$
\left(\begin{array}{c}
d+1+2 \\
2
\end{array}\right)-\operatorname{deg} X=\left(\begin{array}{c}
d+3 \\
2
\end{array}\right)-\left(\begin{array}{c}
d+2 \\
2
\end{array}\right)-h=d+2-h \geq 1 .
$$

If $F=F_{1} F_{2} \in H^{0}\left(\mathscr{I}_{X}(g)\right)$, with $\operatorname{deg} F_{1}=r_{1}>0, \operatorname{deg} F_{2}=r_{2}>0$ and $r_{1}+r_{2}=g$, then, by Proposition 2.2, $F_{1}\left(\right.$ resp. $\left.F_{2}\right)$ can contain at most $\left(\begin{array}{c}r_{1}+2 \\ 2\end{array}\right)-1$ (resp. $\left(\begin{array}{c}r_{2}+2 \\ 2\end{array}\right)-1$ ) points of $X$. So

$$
n \leq\left(\begin{array}{c}
r_{1}+2 \\
2
\end{array}\right)-1+\left(\begin{array}{c}
r_{2}+2 \\
2
\end{array}\right)-1,
$$

from which it follows that

$$
d^{2}+3 d+2 h \leq r_{1}^{2}+r_{2}^{2}+3 g-2 .
$$


But $r_{1}^{2}+r_{2}^{2}=g^{2}-2 r_{1} r_{2}$, where

$$
r_{1} r_{2}=r_{1}\left(g-r_{1}\right) \geq g-1
$$

and so

$$
d^{2}+3 d+2 h \leq g^{2}+g .
$$

If $g \leq d$, then we get a contradiction for any $h$. If $g=d+1$, then we conclude that $h \leq 1$. In this case, not every curve containing $X$ of degree $g=d+1$ is irreducible, but using [11, Theorem 3.5] we see that this is true for the generic one, because there exists one which is nonsingular.

The most important result relating UPP and the plane section of curves is the following:

Theorem 2.4 (Uniform Position Lemma, [7, §2]). If $C \subset \mathbb{P}_{k}^{3}$ is an integral curve and chark $=0$, then the points of the generic plane section of $C$ are in uniform position.

Corollary 2.5. If $C \subset \mathbb{P}_{k}^{3}$ is an integral curve and chark $=0$, the general minimal curve containing the generic plane section of $C$ is irreducible.

Proof. Just apply Proposition 2.3 and Theorem 2.4 .

Example 2.6 (see [14, Example 1.2]). Let $C$ be the curve defined as the complete intersection of $x_{0}{ }^{q}-x_{1} x_{3}{ }^{q-1}=x_{1}{ }^{q}-x_{2} x_{3}{ }^{q-1}=0$, where $q=p^{f}$, for some $f>0$. On the open affine set defined by $x_{3} \neq 0$ the curve $C$ is described by

$$
x_{0}=t, x_{1}=t^{q}, x_{2}=t^{q^{2}}
$$

so that $C$ is rational and integral. However the general plane section of $C$ looks like a 2-dimensional vector space over a field with $q$ elements. Indeed, if $P_{0}, P_{1}, P_{2} \in C$ are linearly independent and $H$ is the plane containing the three points, then the points of $C \cap H$ are given by

$$
P=P_{0}+\lambda_{1}\left(P_{1}-P_{0}\right)+\lambda_{2}\left(P_{2}-P_{0}\right),
$$

where $\lambda_{i}^{q}=\lambda_{i}$ for $i=1,2$. Note also that given two points $P_{0}, P_{1} \in C$, the line $P_{0} P_{1}$ meets $C$ in the points given by

$$
P=P_{0}+\mu\left(P_{1}-P_{0}\right),
$$

where $\mu^{q}=\mu$. So in every generic plane section of $C$ there are, for $q \geq 4$, at least 3 collinear points and 3 linearly independent points. It means that the generic plane section of $C$ does not have the UPP.

Since $C$ is a complete intersection of two surfaces of degree $q, C \cap H$ is a complete intersection, too, and the minimal curves containing $C \cap H$ have degree $q$. Since $C$ is integral, these surfaces are integral and the generic plane section of each of them is an integral curve in $H$. So, in this case, every minimal curve containing the generic plane section of $C$ is irreducible, because every minimal surface containing $C$ is irreducible. 


\section{THE FIBRES OF MORPHISMS}

In this section we recall some results that will be useful in the sequel.

Lemma 3.1. Let $Y$ be a locally noetherian and integral scheme and $f: X \rightarrow Y$ a morphism of finite type. Then there exists an open nonempty subset $U$ of $Y$ such that $\left.f\right|_{f^{-1}(U)}: f^{-1}(U) \rightarrow U$ is flat.

Proof. This follows from [4, Théorème 6.9.1], with $\mathscr{F}=\mathscr{O}_{X}$.

Lemma 3.2 ([4, Corollaire 3.3.5]). Let $X$ and $Y$ be locally noetherian schemes and $f: X \rightarrow Y$ be a flat morphism. If $Y$ is reduced in the points of $f(X)$ and if $f^{-1}(y)$ is a $k(y)$-scheme reduced for every $y \in f(X)$, then $X$ is reduced.

Another result that will be useful in the proof of the main result of this paper is the following:

Theorem 3.3. Let $f: X \rightarrow Y$ be a dominant morphism from an irreducible algebraic variety $X$ to an algebraic variety $Y$. Let $U \subseteq Y$ and $V \subseteq f^{-1}(U) \subset X$ be affine open subsets and $u_{1}, \ldots, u_{r} \in k[V]$ algebraically independent over $k(U)=$ $k(Y)$ such that for some variable $T$,

$$
k[V] \cong k[U]\left[u_{1}, \ldots, u_{r}\right][T] /(F),
$$

with $F \in k[U]\left[u_{1}, \ldots, u_{r}\right][T]$ an irreducible polynomial. If $k(Y)$ is algebraically closed in $k(X)$, there exists a nonempty open subset $W \subset V$ such that for every $y \in W$ the fibre $f^{-1}(y)$ is geometrically integral.

Proof. The proof works as in [15, Theorem 1, p. 139].

\section{Curves of least Degree}

In this section we consider an integral curve $C \subset \mathbb{P}_{k}^{3}$, where $k$ is an algebraically closed field of any characteristic. Let $\left\{\underline{x}_{i}\right\}$ be a system of coordinates over $\mathbb{P}^{3}$ and $\left\{\underline{t}_{j}\right\}$ a system of coordinates over the dual space $\mathbb{P}^{3^{\vee}}$. Denote by $\mathscr{I}_{C}$ the ideal sheaf of $C$ in $\mathbb{P}^{3}$ and let us consider the incidence variety $M \subset \mathbb{P}^{3^{\vee}} \times \mathbb{P}^{3}$ determined by

$$
h=\sum_{i=0}^{3} t_{i} x_{i}=0 .
$$

Let us consider the two projections

$$
\begin{aligned}
& p: M \rightarrow \mathbb{P}^{3}, \\
& g: M \rightarrow \mathbb{P}^{3 \vee}
\end{aligned}
$$

and the following module over $\mathscr{O}_{M}$ :

$$
\mathscr{I}(m, n)=g^{\star}\left(\mathscr{O}_{\mathbb{P}^{3 \vee}}(m)\right) \otimes_{\mathscr{O}_{M}} p^{\star}\left(\mathscr{I}_{C}(n)\right) .
$$

If $T=p^{-1}(C)$, then it is easy to see that $T$ is integral. Indeed, every fibre of the projection $\left.p\right|_{T}: T \rightarrow C$ is integral and equidimensional of dimension 2 , being isomorphic to a projective plane. So $T$ is irreducible and $\left.p\right|_{T}$ is flat, by [8, Ch. III, Theorem 9.9] (note that $T \subset \mathbb{P}^{3^{\vee}} \times C$ ). By Lemma $3.2 T$ is reduced. It is also possible to show that $\mathscr{I}=\mathscr{I}_{T}$, the ideal sheaf of $T$ in $M$ (see [1]). 
Theorem 4.1. If $X \subset \mathbb{P}^{2}$ is a generic plane section of an integral curve $C \subset \mathbb{P}^{3}$, then the generic curve in $\mathbb{P}^{2}$ of minimal degree containing $X$ is irreducible. In particular, if there exists only one curve of minimal degree containing $X$, such a curve is irreducible.

Proof. Let us denote by $s$ the minimal degree of a plane curve containing $X$. Then there exists $\alpha \gg 0$ such that $H^{0}(\mathscr{I}(\alpha, s)) \neq 0$ (see [6] and [1, Proposition 2]). So we can take a general form $F \in H^{0}(M, \mathscr{I}(\alpha, s))$, with $\alpha$ minimal, in such a way that it determines a hypersurface $S$ in the incidence variety $M$. Since $\mathscr{I}=\mathscr{I}_{T}$, $T \subset S . T$ is integral and so $S$ is integral, too, by the minimality of $s$ and $\alpha$.

Since the projection $T \rightarrow \mathbb{P}^{3^{\vee}}$ is dominant, $S \rightarrow \mathbb{P}^{3^{\vee}}$ is dominant, too, and, as in [1, Proposition 2], we see that for a generic $P_{H} \in \mathbb{P}^{3^{\vee}}$ corresponding to a generic plane in $\mathbb{P}^{3}, p\left(S \cap g^{-1}\left(P_{H}\right)\right)$ is a curve of degree $s$ containing $C \cap H$.

Note that by the Künneth formula (see [13, Ch. VI, Corollary 8.13]) we get

$$
H^{1}\left(\mathscr{O}_{\mathbb{P}^{3} \times \mathbb{P}^{3}}(m, n)\right)=0 \forall m, n .
$$

This implies that we have the following surjective map:

$$
H^{0}\left(\mathscr{O}_{\mathbb{P}^{3} \vee} \times \mathbb{P}^{3}(m, n)\right) \rightarrow H^{0}\left(\mathscr{O}_{M}(m, n)\right) \rightarrow 0 \forall m, n .
$$

So, since $\mathscr{I} \subset \mathscr{O}_{M}$, the form $F \in H^{0}(\mathscr{I}(\alpha, s))$ defining $S$ can be lifted to a homogeneous polynomial $f \in k[\underline{t} ; \underline{x}]$ of bidegree $(\alpha, s)$. So $S$ in $\mathbb{P}^{3^{\vee}} \times \mathbb{P}^{3}$ is the complete intersection determined by

$$
\begin{gathered}
f(\underline{t} ; \underline{x})=0, \\
\sum_{i=0}^{3} t_{i} x_{i}=0 .
\end{gathered}
$$

Note that restricting to the open subsets $U \subset \mathbb{P}^{3^{\vee}}$, given by $t_{3} \neq 0$, and $V \subset S$, given by $t_{3} \neq 0, x_{2} \neq 0$, we get

$$
\begin{gathered}
k[U]=k\left[t_{0}, t_{1}, t_{2}\right], \\
k[V]=k\left[t_{0}, t_{1}, t_{2}, x_{0}, x_{1}\right] /(F),
\end{gathered}
$$

where $F\left(t_{0}, t_{1}, t_{2}, x_{0}, x_{1}\right)=f\left(t_{0}, t_{1}, t_{2}, 1 ; x_{0}, x_{1}, 1, t_{0} x_{0}+t_{1} x_{1}+t_{2}\right)$.

We want to show that the generic curve of degree $s$ containing $X$ is irreducible. So we show that the generic fibre of the projection $g_{S}: S \rightarrow \mathbb{P}^{3^{\vee}}$ is irreducible. By Theorem 3.3 we see that it's sufficient to show that $k\left(\mathbb{P}^{3^{\vee}}\right)$ is algebraically closed in $k(S)$, since by (4.1) we see that in this case all the conditions are satisfied.

Let us consider the algebraic closure $K$ of $g_{S^{\star}} k\left(\mathbb{P}^{3^{\vee}}\right)$ in $k(S)$. We want to show that $K=g_{S}{ }^{\star} k\left(\mathbb{P}^{3^{\vee}}\right)$. Of course $K$ is finitely generated over $k$ and so it corresponds to an irreducible variety $Z$ such that $k(Z)=K$. Since $K$ is algebraic over $g_{S}{ }^{\star} k\left(\mathbb{P}^{3^{\vee}}\right)$, we see that $\operatorname{dim} Z=3$ and that we can factor $g_{S}$ as a composition of rational maps

$$
S_{\stackrel{\pi_{1}}{\pi_{1}} \rightarrow}^{\stackrel{\pi_{2}}{\rightarrow}} \mathbb{P}^{3 \vee}
$$

where $\pi_{1}$ is dominant and has irreducible fibres (by Theorem 3.3 ) and $\pi_{2}$ is generically finite.

Let us take an open subset $U \subset \mathbb{P}^{3^{\vee}}$, whose points correspond to planes that are general for $C$, and $V=\pi_{2}{ }^{-1}(U) \subset Z$ and $W=g_{S}{ }^{-1}(U) \subset S$, so that

$$
W \stackrel{\pi_{1}}{\rightarrow} V \stackrel{\pi_{2}}{\rightarrow} U
$$


is a factorisation of $g_{U}: W \rightarrow U$ and the following conditions are satisfied:

$g_{U}$ is flat, the projection $T \cap\left(g^{-1}(U)\right) \rightarrow U$ is flat, with $g: M \rightarrow \mathbb{P}^{3^{\vee}}$, $g_{U}^{-1}\left(P_{H}\right)$ is reduced and $\operatorname{dim} g_{U}^{-1}\left(P_{H}\right)=1$ for any $P_{H} \in U$

and

$$
\pi_{2}^{-1}(L \cap U) \text { is irreducible }
$$

for any $L \subset \mathbb{P}^{3^{\vee}}$ plane such that $L \cap U \neq \emptyset$. Note that $U \neq \emptyset$ by Lemma 3.1 and by [9, I, Theorem $6.10(3)]$.

We will show that $\pi_{2}: V \rightarrow U$ is an isomorphism.

Let us consider the fibred product $W \times_{U} V$ and the projection

$$
\phi: W \times_{U} V \rightarrow V .
$$

Claim 1. $W \times_{U} V$ is equidimensional of dimension 4 and is reduced.

Proof of Claim 1. By (4.3) any point $Q_{0} \in V$ is such that $\pi_{2}\left(Q_{0}\right)=P_{H} \in U \subset \mathbb{P}^{3 \vee}$ corresponds to a plane $H$ and so

$$
\phi^{-1}\left(Q_{0}\right)=\left(W \times_{U} V\right) \times_{V} \operatorname{Spec} k\left(Q_{0}\right) \cong W \times_{U} \operatorname{Spec} k\left(P_{H}\right)=g_{U}^{-1}\left(P_{H}\right) .
$$

So $\phi^{-1}\left(Q_{0}\right)$ is isomorphic to a curve in $H$ of degree $s$ containing $C \cap H$. So all the fibres of $\phi$ are equidimensional of dimension 1 , and we see that $W \times_{U} V$ is equidimensional of dimension 4. $\phi^{-1}\left(Q_{0}\right)$ is also reduced for any $Q_{0} \in V$ and $\phi$ is flat, by base change, being $g_{U}$ flat by construction. So using Lemma 3.2 we see that $W \times_{U} V$ is reduced.

Let us now consider $g: M \rightarrow \mathbb{P}^{3^{\vee}}$ and $T=p^{-1}(C)$. Since $g^{-1}(U)$ is an open subset of $M$ and $T \subset M$, then $T \cap\left(g^{-1}(U)\right)$ is dense in $T$. So we can consider $T^{\prime}=\left(T \cap\left(g^{-1}(U)\right)\right) \times_{U} V$.

Claim 2. $T^{\prime}$ is integral.

Proof of Claim 2. Let us consider the projection

$$
\psi: T^{\prime} \rightarrow C
$$

so that $\psi\left(T^{\prime}\right)$ is an open subset of $C$. Taken any $P_{0} \in \psi\left(T^{\prime}\right)$, we see that

$$
\psi^{-1}\left(P_{0}\right) \cong\left(L_{P_{0}} \cap U\right) \times_{U} V \cong \pi_{2}^{-1}\left(L_{P_{0}} \cap U\right),
$$

where $L_{P_{0}} \subset \mathbb{P}^{3}$ is the linear subvariety of the planes containing $P_{0}$ and has dimension 2. By the definition of $T^{\prime}$ we see that $L_{P_{0}} \cap U \neq \emptyset$ and by (4.4) $\pi_{2}{ }^{-1}\left(L_{P_{0}} \cap U\right)$ is irreducible for any $P_{0} \in \psi\left(T^{\prime}\right)$. So from (4.6) we conclude that $T^{\prime}$ is irreducible.

Let us now consider $\phi_{T^{\prime}}: T^{\prime} \rightarrow V$. This is a surjective morphism such that

$$
\phi_{T^{\prime}}{ }^{-1}\left(Q_{0}\right)=T^{\prime} \times_{V} \operatorname{Spec} k\left(Q_{0}\right) \cong T \times_{U} \operatorname{Spec} k\left(P_{H}\right) \cong C \cap H .
$$

Since by construction $T \cap\left(g^{-1}(U)\right) \rightarrow U$ is flat, by base change $\phi_{T^{\prime}}$ is flat, too, and so by Lemma 3.2 we see that $T^{\prime}$ is reduced. So the claim is proved.

Claim 3. $W \times_{U} V$ is integral. 
Proof of Claim 3. Since $\operatorname{dim} W \times_{U} V=4$ and $W \times_{U} V$ is reduced, there exists an integral component $\tilde{W}$ of $W \times_{U} V$, with $\operatorname{dim} \tilde{W}=4$, such that $T^{\prime} \subset \tilde{W}$. Since $\phi_{T^{\prime}}$ is surjective, then

$$
\varphi: \tilde{W} \rightarrow V
$$

is surjective too. So, taken any $Q_{0} \in V$, if $\pi_{2}\left(Q_{0}\right)=P_{H}$, we have

$$
\operatorname{dim} \varphi^{-1}\left(Q_{0}\right) \geq 1
$$

and

$$
C \cap H \cong \phi_{T^{\prime}}^{-1}\left(Q_{0}\right) \subset \varphi^{-1}\left(Q_{0}\right) \subset \phi^{-1}\left(Q_{0}\right)=g_{U}{ }^{-1}\left(P_{H}\right)
$$

by (4.5) and (4.7). By (4.3) $\phi^{-1}\left(Q_{0}\right)=g_{U}^{-1}\left(P_{H}\right)$ is equidimensional of dimension 1 for any $Q_{0} \in V$ and we see by (4.8) that $\varphi^{-1}\left(Q_{0}\right) \subset g_{U}^{-1}\left(P_{H}\right)$ is equidimensional of dimension 1 for any $Q_{0} \in V$. So $\varphi^{-1}\left(Q_{0}\right)$ is a component of the curve $g_{U}^{-1}\left(P_{H}\right)$ of degree $s$ containing $C \cap H$ by (4.9). By the minimality of $s, \varphi^{-1}\left(Q_{0}\right) \cong g_{U}{ }^{-1}\left(P_{H}\right)=$ $\phi^{-1}\left(Q_{0}\right)$ for any $Q_{0} \in V$. So $\tilde{W}=W \times_{U} V$, which means that $W \times_{U} V$ is reduced and irreducible.

Claim 4. $W \times_{U} V=W$.

Proof of Claim 4. Let us now consider the projection $\psi: W \times_{U} V \rightarrow W$ and take any $P_{0} \in W$. Then

$$
\psi^{-1}\left(P_{0}\right)=\operatorname{Spec} k\left(P_{0}\right) \times_{U} V \cong \pi_{2}^{-1}\left(g\left(P_{0}\right)\right) .
$$

So $\psi$ is a finite morphism and

$$
\psi^{\star} k(W) \subset k\left(W \times_{U} V\right)
$$

is an algebraic field extension. Obviously we have the following commutative diagram:

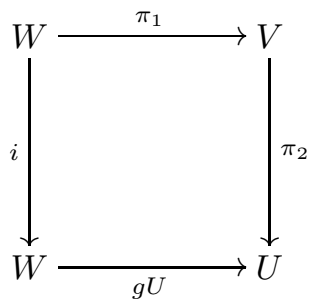

and so, by the properties of the fibred product, there exists a morphism $\tau: W \rightarrow$ $W \times_{U} V$ that factors the identity on $W$ :

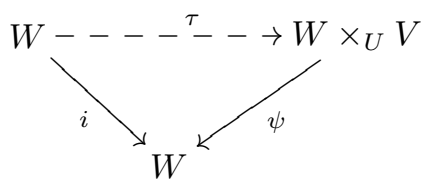

So, being $W \times_{U} V$ integral, $\tau$ must be surjective, and so we get the inclusion $\tau^{\star} k\left(W \times_{U} V\right) \subset k(W)$. Applying $\tau^{\star}$ to (4.10) we get

$$
k(W)=\tau^{\star} \psi^{\star} k(W) \subset \tau^{\star} k\left(W \times_{U} V\right) \subset k(W)
$$

so that $\tau^{\star} k\left(W \times_{U} V\right)=k(W)$. It means that $\tau$ is an isomorphism, and from (4.11) we see that $\psi$ is an isomorphism.

From $W=W \times_{U} V$ it follows immediately that $U=V$, and in this way the theorem is proved. 
Now we show that from this result it follows easily that the Hilbert function of the generic plane section of $C$ is of decreasing type. Let $X=C \cap H$ be a general plane section of $C$ and $H\left(X,,_{-}\right)$the Hilbert function of $X$ in $H$. The first difference of the Hilbert function is

$$
\Delta H(X, i)=H(X, i)-H(X, i-1) \forall i .
$$

It is known [3] that there exists $a_{1} \leq a_{2} \leq t$ such that

$$
\Delta H(X, i)= \begin{cases}i+1 & \text { for } i=0, \ldots, a_{1}-1, \\ a_{1} & \text { for } i=a_{1}, \ldots, a_{2}-1, \\ <a_{1} & \text { for } i=a_{2}, \\ \text { nonincreasing } & \text { for } i=a_{2}, \ldots, t, \\ 0 & \text { for } i>t .\end{cases}
$$

Definition 4.2. We say that $X$ has the Hilbert function of decreasing type if for $a_{2} \leq i<j<t$ we have $\Delta H(X, i)>\Delta H(X, j)$.

It is well known that the Hilbert function of the generic plane section of an integral curve is of decreasing type (see [7, 12, [5, Lemme 3.2] for chark $=0$ and [1, Theorem 3.3] for any characteristic). This fact is a direct consequence of Theorem 4.1 ;

Corollary 4.3. Let $C \subset \mathbb{P}^{3}$ be an integral curve and let $X=C \cap H$ be a general plane section of $C$. Then the Hilbert function of $X$ is of decreasing type.

Proof. Let $c_{i}=\Delta H(X, i)$ for each $i$. Let us suppose that for some $s \geq a_{1}$ we have $c_{s-1}=c_{s}$. From [10, Theorem 2.9] we see that the curves in $H$ of degree $s$ containing $X$ have a common factor $D$ of degree $c_{s}$. In particular $D$ is a common factor of the minimal curves containing $X$. However, from Theorem 4.1 the general minimal curve is irreducible. So $D$ is irreducible and is the only minimal curve containing $X$. Since $\operatorname{deg} D=c_{s}$, we have $c_{s}=a_{1}$ and so the Hilbert function of $X$ is of decreasing type.

\section{ACKNOWLEDGEMENTS}

The author would like to thank her advisor, Rosario Strano, for all his useful suggestions and Riccardo Re for many helpful conversations.

\section{REFERENCES}

1. P. Bonacini, Hilbert functions of decreasing type in positive characteristic, to appear in Rend. Sem. Mat. Univ. Pol. Torino.

2. A. V. Geramita, P. Maroscia, The ideal of forms vanishing at a finite set of points in $\mathbb{P}^{n}, \mathrm{~J}$. Alg. 90 (1984), 528-555. MR760027 (86e:14025)

3. A. V. Geramita, P. Maroscia, L. G. Roberts, The Hilbert function of a reduced k-algebra, J. London Math. Soc. (2) 28 (1983), 443-452. MR724713 (85c:13018)

4. A. Grothendieck, Éléments de géométrie algébrique: IV. Étude locale de schémas et des morphismes de schémas, Seconde Partie, Publ. Math. IHES 24, 1965.

5. L. Gruson, C. Peskine, Genre de courbes de l'espace projectif, Algebraic geometry (Proc. Sympos., Univ. Troms $\varnothing$, Troms $\varnothing, 1977)$, pp. 31-59, Lecture Notes in Math. 687, Springer, Berlin, 1978. MR 0527229 (81e:14019)

6. L. Gruson, C. Peskine, Section plane d'une courbe gauche: postulation, Progress in Math. 24, Birkhäuser, Boston, MA (1982), 33-35. MR0685762 (84c:14045)

7. J. Harris, The genus of space curves, Math. Ann. 249 (1980), 191-204. MR579101|(81i:14022) 
8. R. Hartshorne, Algebraic Geometry, Graduate Texts in Mathematics 52, Springer-Verlag, New York-Heidelberg, 1977. MR0463157 (57:3116)

9. J.-P. Jouanolou, Théorèmes de Bertini et Applications, Progress in Mathematics 42, Birkhäuser Boston, Boston, MA, 1983. MR725671 (86b:13007)

10. R. Maggioni, A. Ragusa, Connections between Hilbert function and geometric properties for a finite set of points in $\mathbb{P}^{2}$, Matematiche (Catania) 39 (1984), 153-170. MR.934156 (89c:14046)

11. R. Maggioni, A. Ragusa, Nonsingular curves passing through points of $\mathbb{P}^{2}$ in generic position, I, J. Alg. 92 (1985), 176-193. MR772479 (86c:14026)

12. R. Maggioni, A. Ragusa, The Hilbert function of generic plane sections of curves in $\mathbb{P}^{3}$, Inv. Math. 91 (1988), 253-258. MR 922800 (89g:14027)

13. J. S. Milne, Étale cohomology, Princeton Mathematical Series 33, Princeton University Press, Princeton, NJ, 1980. MR.559531 (81j:14002)

14. J. Rathmann, The uniform position lemma for curves in characteristic $p$, Math. Ann. 276 (1987), 565-579. MR:879536 (89g:14026)

15. I. R. Shafarevich, Basic Algebraic Geometry I-Varieties in Projective Space, 2nd rev. and exp. ed., Springer-Verlag, Berlin-Heidelberg, 1994. MR1328833 (95m:14001)

Dipartimento di Matematica e Informatica, Università degli Studi di Catania, Viale A. Doria 6, 95125 Catania, Italy

E-mail address: bonacini@dmi.unict.it 